\title{
Rapid Determination of Swiss Cheese Composition by Fourier Transform Infrared/Attenuated Total Reflectance Spectroscopy
}

\author{
L. E. Rodriguez-Saona, ${ }^{\star}$ N. Koca, ${ }^{\star} \dagger$ W. J. Harper, ${ }^{\star}$ and V. B. Alvarez ${ }^{\star 1}$ \\ *Department of Food Science and Technology, The Ohio State University, \\ 110 Parker Food Science and Technology Building, Columbus 43210-1007 \\ †Department of Food Engineering, Ege University, Izmir, Turkey
}

\section{ABSTRACT}

There is a need for rapid and simple techniques that can be used to predict the quality of cheese. The aim of this research was to develop a simple and rapid screening tool for monitoring Swiss cheese composition by using Fourier transform infrared spectroscopy. Twenty Swiss cheese samples from different manufacturers and degree of maturity were evaluated. Direct measurements of Swiss cheese slices $(\sim 0.5 \mathrm{~g})$ were made using a MIRacle 3-reflection diamond attenuated total reflectance (ATR) accessory. Reference methods for moisture (vacuum oven), protein content (Kjeldahl), and fat (Babcock) were used. Calibration models were developed based on a cross-validated (leave-one-out approach) partial least squares regression. The information-rich infrared spectral range for Swiss cheese samples was from 3,000 to $2,800 \mathrm{~cm}^{-1}$ and 1,800 to 900 $\mathrm{cm}^{-1}$. The performance statistics for cross-validated models gave estimates for standard error of cross-validation of $0.45,0.25$, and $0.21 \%$ for moisture, protein, and fat respectively, and correlation coefficients $\mathrm{r}>$ 0.96. Furthermore, the ATR infrared protocol allowed for the classification of cheeses according to manufacturer and aging based on unique spectral information, especially of carbonyl groups, probably due to their distinctive lipid composition. Attenuated total reflectance infrared spectroscopy allowed for the rapid ( $\sim 3-\mathrm{min}$ analysis time) and accurate analysis of the composition of Swiss cheese. This technique could contribute to the development of simple and rapid protocols for monitoring complex biochemical changes, and predicting the final quality of the cheese.

Key words: Swiss cheese, composition, attenuated total reflectance infrared spectroscopy, multivariate analysis

Received October 5, 2005.

Accepted December 1, 2005.

${ }^{1}$ Corresponding author: alvarez.23@osu.edu

\section{INTRODUCTION}

Current methods for analyzing cheese composition are time-consuming, expensive, and require the use of hazardous organic solvents. Infrared (IR) spectroscopy is a rapid, inexpensive, and sensitive technology used for the high-throughput analysis of food components without requiring special skills from the users. Advances in Fourier transform infrared (FTIR) instrumentation and pattern recognition techniques have made it possible to extract information related to composition and conformation of food components from the spectra (Udelhoven et al., 2000). Mid-IR spectroscopy $\left(4,000\right.$ to $\left.700 \mathrm{~cm}^{-1}\right)$ has much to offer the analyst because specific bands may be assigned to specific chemical entities, and because it provides narrow bands arising from group vibrations with known assignment in most cases. The mid-IR spectra allow for the chemically based discrimination of organic constituents, and produce distinct and reproducible biochemical fingerprints.

Near infrared and mid-IR spectroscopy have been extensively used for the analysis of several milk components such as protein, fat, moisture, sugars, casein, cholesterol, and contaminants such as tetracycline (Rodriguez-Otero et al., 1997; O'Sullivan et al., 1999; Tsenkova et al., 1999; Luginbühl, 2002; Paradkar and Irudayaraj, 2002; Sivakesava and Irudayaraj, 2002; Sorensen et al., 2003). Near-infrared spectroscopy has been widely studied for the rapid determination of major cheese constituents such as protein, fat, moisture, DM, and total solids (Frank and Birth, 1982; Wehling and Pierce, 1988; Pierce and Wehling, 1994; Rodriguez-Otero et al., 1995; McQueen et al., 1995; Wittrup and Noøgaard, 1998; Adamopoulos et al., 2001; Čurda and Kukačková, 2004). On the contrary, few studies have reported the application of midIR spectroscopy for cheese analysis because of difficulties in the sampling procedures due to the lack of homogeneity of the components in the cheese (McQueen et al., 1995). Chen et al. (1998) investigated the application of FTIR spectroscopy to examine the changes during ripening for Cheddar cheeses with different fat levels, and reported dis- 
tinct changes in spectral bands of fat and protein during ripening that could provide rapid characterization and determination of the age of the cheese undergoing ripening.

Infrared spectroscopy has also found applications for authentication of products by commodity, variety, and geographic origin. The potential of IR spectroscopy for monitoring the geographic origin of cheeses has been shown (Pillonel et al., 2003; Karoui et al., 2004, 2005), allowing classification of samples according to the region of cheese origin. Picque et al. (2002) reported the discrimination of Emmental cheeses from different regions by IR spectroscopy. The authors highlighted the ability of IR to discriminate according to the nature of the milk used for the manufacturing process.

The objective of this research was to investigate the use of FTIR/attenuated total reflectance (ATR) spectroscopy for measurement of fat, protein, and moisture content in Swiss cheese samples.

\section{MATERIALS AND METHODS}

\section{Cheese Sampling}

Nineteen Swiss cheese samples were supplied by 3 Swiss cheese manufacturers in Ohio and an additional Swiss cheese sample was purchased at a local store. Swiss cheese obtained from Ohio manufacturers included samples taken from different production dates and at aging times that ranged from 30 to $>340 \mathrm{~d}$. Cheese samples were vacuum-packaged and stored at refrigerated $\left(4^{\circ} \mathrm{C}\right)$ conditions until analysis.

\section{Reference Analysis}

The percentage compositions of moisture, fat, and protein were determined using the reference methods according to Bradley et al. (1992). All samples were tested in duplicate. The fat content was determined using the Babcock method, the moisture content by vacuum-oven method, and the protein content by Kjeldahl method.

\section{FTIR/ATR Spectroscopy Measurements}

Infrared spectra were recorded between 4,000 and $700 \mathrm{~cm}^{-1}$ at a resolution of $4 \mathrm{~cm}^{-1}$ on a FTS Excalibur 3500GX FTIR spectrometer (Digilab, Randolph, MA) mounted with an MIRacle ATR accessory equipped with a high pressure clamp (Pike Technologies, Madison, WI). The instrument has a computer-controlled dualsource assembly with Permaglow mid-IR source and extended range $\mathrm{KBr}$ beam splitter. The diamond MIRacle ATR crystal utilizes a $0.5-\mathrm{mm}$ Type $2 \mathrm{~A}$ diamond. A 3 -reflection diamond crystal provides an increase in sample absorbance allowing low concentration components to be measured.

Cheese slices $(\sim 0.5 \mathrm{~g})$ were taken from the center of the cheese avoiding the eyes. The cheese slices were pressed with the high-pressure clamp to ensure good contact between the sample and the diamond crystal. To improve the signal to noise ratio, 128 scans were coadded for each spectrum. The absorbance spectrum was obtained by rationing the single beam spectrum against that of the air background. Four spectra were recorded for each sample using pieces taken from different areas of Swiss cheese sample.

\section{Multivariate Data Analysis}

Partial least squares regression (PLSR) was applied to generate calibration models using Pirouette pattern recognition software (Version 3.02 for Windows NT, Infometrix, Inc., Woodinville, WA). The optimum number of latent variables used for prediction was determined by full cross-validation (leave-one-out approach). The performance of the resulting models was evaluated in terms of latent variables, cumulative variance, standard error of calibration (SEC), standard error of cross validation (SECV), and coefficient of determination ( $r$ ) for the calibration models by using reference data. The model producing the first local minimum SECV was selected as the best model for the spectral data set (Haaland and Thomas, 1988; Martens and Naes, 1989). Classification models were developed using Soft Independent Modeling of Class Analogies (SIMCA). The scores plot (a projection of the original data onto the principal component axes) was used for the visualization of clustering among samples (sample patterns, groupings or outliers).

\section{RESULTS AND DISCUSSION}

The application of a mid-IR ATR accessory equipped with a multiple-reflection diamond crystal provided a 3 -fold increase in sample response compared with the standard single reflection crystal plate (Pike Technologies, 2005) and allowed for the generation of reproducible, high-quality IR spectra. Figure 1A shows the ATRIR spectra of Swiss cheese samples, indicating some of the spectral bands arising from specific functional group vibrations. The spectrum shows typical bands arising from amide (1,650 to $\left.1,540 \mathrm{~cm}^{-1}\right)$ and lipid (1,765 to $1,730 \mathrm{~cm}^{-1}$ and 3,000 to $2,800 \mathrm{~cm}^{-1}$ ) groups. Strong and broad absorption bands of water are shown in the region of 3,700 to $3,000 \mathrm{~cm}^{-1}$. Water also absorbs in the region of 1,700 to $1,600 \mathrm{~cm}^{-1}$, which can affect the amide I signal (Chen et al., 1998; Chen and Irudayaraj, 1998). Dufour et al. (2000) reported that the triacylglycerols 

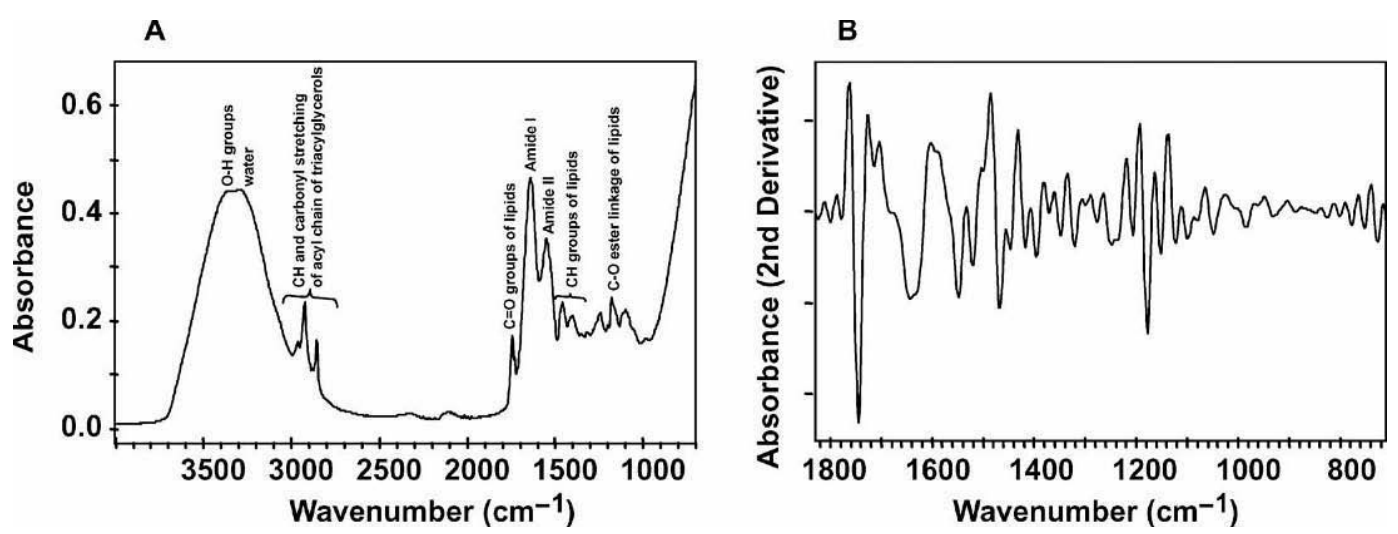

Figure 1. Midinfrared spectra of Swiss cheese using an attenuated total reflectance accessory equipped with a 3-bounce diamond plate. A) Midinfrared absorbance spectrum collected in the 4,000 to $700 \mathrm{~cm}^{-1}$ range; B) Second-derivative (5-point window) spectral transformation collected in the 1,800 to $700 \mathrm{~cm}^{-1}$ range.

ester linkage $\mathrm{C}-\mathrm{O}\left(\sim 1,175 \mathrm{~cm}^{-1}\right), \mathrm{C}=\mathrm{O}\left(\sim 1,750 \mathrm{~cm}^{-1}\right)$ group, and acyl chain $\mathrm{C}-\mathrm{H}\left(3,000\right.$ to $\left.2,800 \mathrm{~cm}^{-1}\right)$ stretch vibrations are commonly used to determine fat. Chen et al. (1998) also reported fat-related bands occurring at 1,477 to $1,400 \mathrm{~cm}^{-1}$ (C-H bending), $1,240 \mathrm{~cm}^{-1}$, and 1,170 to $1,115 \mathrm{~cm}^{-1}$ (C-O stretching), which were also observed in the cheese spectra. The Savitzky-Golay second-derivative transform (Figure 1B) allowed the extraction of useful band information through the removal of baseline variations and resolution of overlapping peaks (Hrushka, 1987).

Calibration models for the determination of moisture, protein, and fat content in Swiss cheese samples showed good correlations between the specified component levels and the ATR-IR estimated concentrations (Figure 2) with correlation coefficients $>0.96$. The crossvalidated (leave-one-out) PLSR model results are shown in Table 1. Second-derivative transformations (5 points window size) of the spectral data enhanced the prediction ability of the models and provided the best performance statistics with SECV, an estimate of the magnitude of error expected when independent samples are predicted using the model, of $0.21 \%$ for fat, $0.25 \%$ for protein, and $0.45 \%$ for moisture. A major advantage of the application of IR spectroscopy is its ability to rapidly scan spectra directly on the cheese sample and obtain a fingerprint spectrum on a given cheese that contains information about its physicochemical characteristics. One limitation of the application of mid-IR techniques in cheese analysis is the fact that cheeses are not spatially homogeneous, showing differences in composition at different parts of the same sample (McQueen et al., 1995). Other sources of variability include morphological changes during aging (McQueen et al., 1995), nonhomogeneity of fat globules, bound moisture in the protein matrix, and the presence of voids in the cheese matrix (Chen and Irudayaraj, 1998). McQueen et al. (1995) evaluated the application of a horizontal ZnSe ATR accessory and reported standard error of predictions (SEP) between 4 and $9 \%$ for moisture, protein, and fat content with low correlation coefficients $(0.64$ to 0.92$)$. The authors attributed the poor performance of the ATR-IR technique to heterogeneity in the cheeses resulting from adsorption of the fat droplets to the ZnSe crystal. The use of a diamond crystal provided a chemically inert surface with higher refractive index and IR throughput than ZnSe crystal plates (Pike Technologies, 2005). Furthermore, the application of a 3-bounce ATR accessory showed improved prediction performances of the models, limiting the variance due to sample heterogeneity.

Swiss cheese is a complex matrix that, besides compositional and morphological heterogeneity, contains eyes that introduce further interfering variability to the IR spectral data. There are no published data on the application of IR techniques for the determination of Swiss cheese composition. Reflectance near-infrared spectroscopy has been reported for the determination of chemical constituents in varieties of hard and semihard cheeses. Similar performance statistics were reported for near-IR with $\mathrm{R}^{2}>0.92$ and SEP values ranging from 0.3 to $0.6 \%$ for moisture, 0.3 to $0.5 \%$ for fat, and 0.3 to $0.5 \%$ for protein, depending on the type of cheese and mathematical transformation of the spectral data (Pierce and Wehling, 1994; Rodriguez-Otero et al., 1995; Sorensen and Jepsen, 1998; Wittrup and Nørgaard, 1998; Mazerolles et al., 2000). Improved prediction results have been reported for near-IR analysis of processed cheeses, with SEP of 1.25 and $1.24 \mathrm{~g} / \mathrm{kg}$ for moisture and fat, respectively, probably due to the less heterogeneous composition of the cheese material (Adams et al., 1999). 

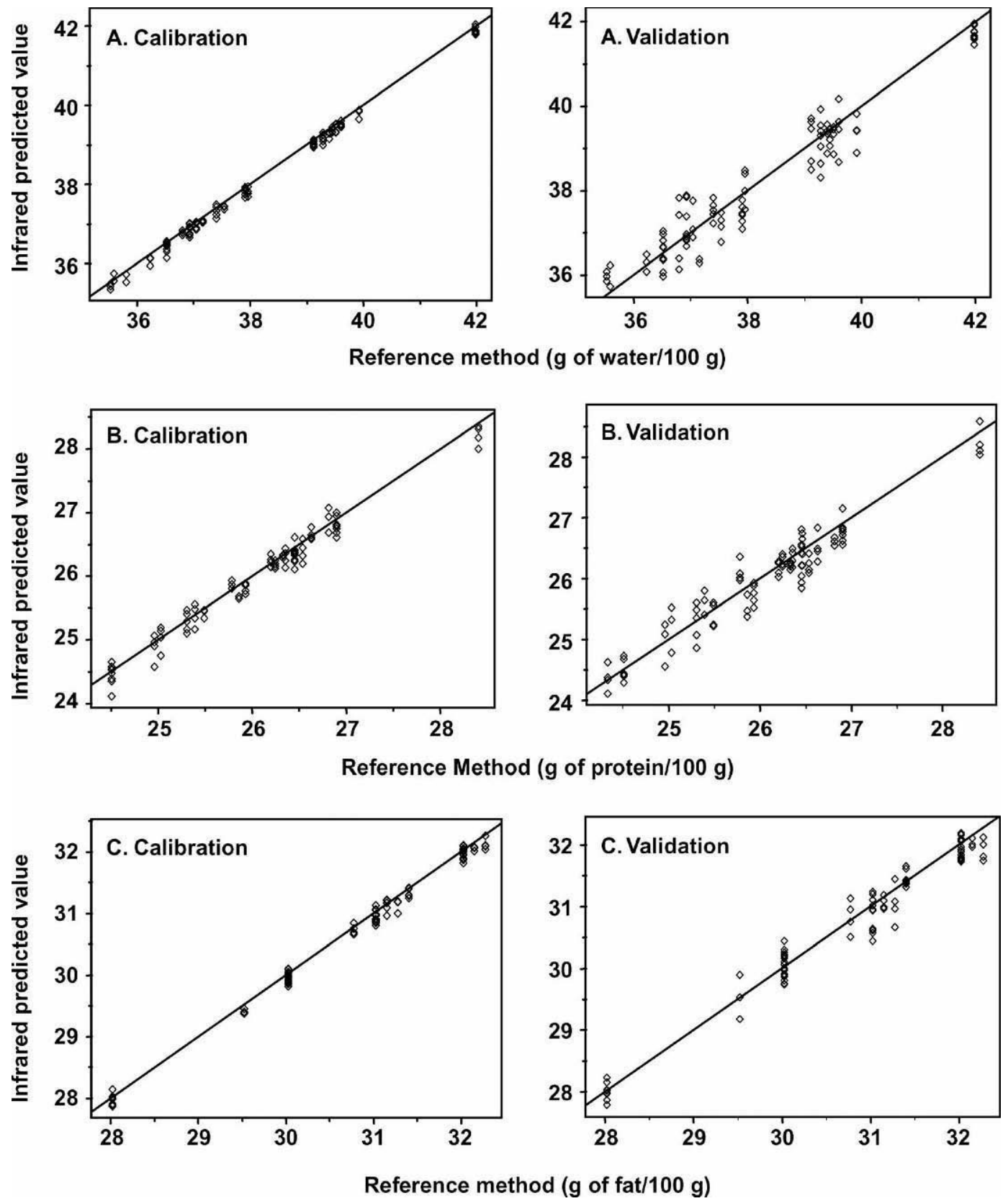

Figure 2. Partial least squares regression (PLSR) calibration and validation plots for A) moisture, B) protein, and C) fat determination in Swiss cheese samples. Second-derivative $\left(\delta^{2} \mathrm{~A} / \delta \lambda^{2}\right)$ spectral transformation was used for the multivariate analysis.

It was possible to classify different Swiss cheese samples using mid-IR technology based on their spectral information (Figure 3). Using the spectral range of 1,800 to $1,200 \mathrm{~cm}^{-1}$, cheese samples from different manufacturers and aging were grouped in well-separated clusters by the SIMCA model. The discrimination analysis (Figure 3B) used to determine the frequencies that accounted for most of the variance among samples, showed that the spectral region from 1,800 to 1,700 $\mathrm{cm}^{-1}$ had an important role in the classification of these Swiss cheese samples. The strong bands arising between 1,765 and $1,730 \mathrm{~cm}^{-1}$ have been correlated with the characteristic signal from the carbonyl group of various $\mathrm{R}(\mathrm{CO}) \mathrm{OH}$ or $\mathrm{R}(\mathrm{CO}) \mathrm{OR}$ (Chen et al., 1998). The changes in carbonyl composition during aging have been attributed to enzymatic lipolysis and proteolysis processes (Chen et al., 1998). Other spectral features involved in the classification were associated with the 
Table 1. Calibration and cross-validation results for Swiss cheese compositional analysis using attenuate total reflectance infrared (IR) spectroscopy ${ }^{1,2}$

\begin{tabular}{llllllll}
\hline Component & $\begin{array}{l}\text { IR frequency } \\
\left(\mathrm{cm}^{-1}\right)\end{array}$ & $\begin{array}{l}\text { Latent } \\
\text { variables }\end{array}$ & $\begin{array}{l}\text { Cumulative } \\
\text { variance }\end{array}$ & SEC & rCal & SECV & rVal \\
\hline Moisture & 3,000 to 2,$800 ; 1,800$ to 900 & 7 & 90.5 & 0.24 & 0.99 & 0.45 & 0.96 \\
Protein & 1,800 to 900 & 9 & 91.6 & 0.16 & 0.99 & 0.25 & 0.96 \\
Fat & 3,000 to 2,$800 ; 1,800$ to 900 & 6 & 84.9 & 0.16 & 0.99 & 0.21 & 0.98 \\
\hline
\end{tabular}

${ }^{1}$ Latent variables $=$ orthogonal factors that provide maximum correlation with dependent variable.

${ }^{2} \mathrm{SEC}=$ Standard error of calibration; $\mathrm{rCal}=$ coefficient of correlation for calibration model; $\mathrm{SECV}=$ standard error of cross-validation; and rVal = coefficient of correlation for validation model.

mid-IR region from 1,500 to $1,200 \mathrm{~cm}^{-1}$ that contained bending modes of $\mathrm{O}-\mathrm{C}-\mathrm{H}, \mathrm{C}-\mathrm{C}-\mathrm{H}$, and $\mathrm{C}-\mathrm{O}-\mathrm{H}$ groups (Paradkar and Irudayaraj, 2002; Sivakesava and Irudayaraj, 2002). Our data did not show discrimination based on spectral patterns associated with $\mathrm{C}-\mathrm{H}$ bonds of methyl and methylene groups of fatty acids $(3,000$ to $2,800 \mathrm{~cm}^{-1}$ ), the amide (1 698 to $1540 \mathrm{~cm}^{-1}$ ), or fingerprint $\left(1,200\right.$ to $\left.900 \mathrm{~cm}^{-1}\right)$ spectral regions. It is important to emphasize that the discrimination of the different Swiss cheese samples was due to the presence of specific marker groups formed during the aging process, such as carbonyl or ester compounds, and not to the major components in cheese (i.e., fat or protein content). Cheese is a complex food matrix in which final quality will be influenced by factors such as the composition of the milk (i.e., moisture, protein and fat level), starter cultures, the manufacturing process, and the biochemical events that occur during ripening of the cheese (Chen et al., 1998). Our results showed that midIR spectroscopy produces specific spectral profiles that may be used for monitoring the biochemical changes occurring during the aging process, and be used to discriminate among different commercial Swiss cheeses to determine factory of origin. Due to the limited Swiss cheese samples with detailed aging information, further research is being undertaken to monitor the complex biochemical changes occurring during the cheeseaging process under controlled manufacturing conditions. This will allow the development of simple and rapid protocols by which cheese can be classified and whereby uniform cheese quality can be established, saving time and money to the cheese industry.

\section{CONCLUSIONS}

Generation of strong, well-separated, and reproducible IR spectral information by using an ATR accessory equipped with a multiple reflection diamond crystal allowed for the accurate determination of the composition and ripening age of Swiss cheese samples. A simple
(A)

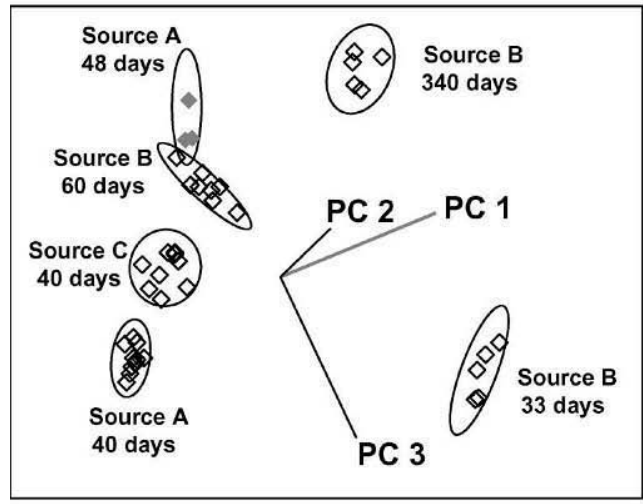

(B)

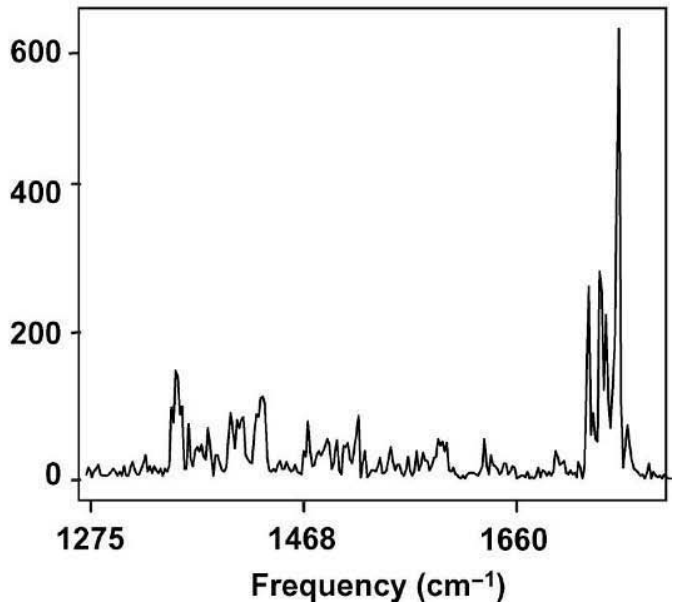

Figure 3. A) Soft independent modeling of class analogy (SIMCA) class projections of transformed (second derivative, 5-point window) midinfrared spectra of Swiss cheese samples with known degree of maturation; B) Discriminative power between Swiss cheese samples based on the SIMCA model. The data comprised repeated observations (3 to 4) collected from cheese slices ( $\sim 0.5 \mathrm{~g})$. Samples from different production lots with different aging times were supplied by local (Ohio) Swiss cheese manufacturers (sources A, B, and C). 
ATR-IR protocol minimized the sample heterogeneity effects of Swiss cheeses that gave predictive models with high correlation coefficients $(\mathrm{r}>0.95)$ and low SECV values for moisture $(0.5 \%)$, protein $(0.3 \%)$, and fat $(0.2 \%)$ determination, regardless of the degree of ripening of the cheese samples. In addition, the ability of IR spectroscopy to provide distinctive bands allowed for the classification of the Swiss cheeses according to their manufacturer and age, presumably due the formation of carbonyl groups $\left(1,800\right.$ to $\left.1,730 \mathrm{~cm}^{-1}\right)$ during cheese ripening. This technique could provide information to further the understanding of the complex biochemical changes occurring during the aging process and contribute to the development of simple and rapid protocols for monitoring the chemical composition and aging changes of Swiss cheese.

\section{ACKNOWLEDGMENTS}

The authors would like to acknowledge the Center for Innovative Food Technology (CIFT) for their financial support. We also would like to thank The Ohio State University Food Industries Center personnel for their collaboration and assistance.

\section{REFERENCES}

Adamopoulos, K. G., A. M. Goula, and H. J. Petropakis. 2001. Quality control during processing of feta cheese-NIR Application. J. Food Compost. Anal. 14:431-440.

Adams, M. J., K. Latham, N. W. Barnett, and A. J. Poynton. 1999. Calibration models for determining moisture and fat content of processed cheese using near-infrared spectrometry. J. Sci. Food Agric. 79:1232-1236.

Bradley, R. L., Jr., E. Arnold, Jr., D. M. Barbano, R. G. Semerad, D. E. Smith, and B. K. Vines. 1992. Chemical and Physical Methods. Pages 433-531 in Standard Methods for the Examination of Dairy Products. 16th ed. R. T. Marshall, ed. American Public Health Association, Washington, DC.

Chen, M., and J. Irudayaraj. 1998. Sampling technique for cheese analysis by FT-IR spectroscopy. J. Food Sci. 63:96-99.

Chen, M., J. Irudayaraj, and D. J. McMahon. 1998. Examination of full fat and reduced fat cheddar cheese during ripening by Fourier transform infrared spectroscopy. J. Dairy Sci. 81:2791-2797.

Čurda, L., and O. Kukačková. 2004. NIR spectroscopy: A useful tool for rapid monitoring of processed cheeses manufacture. J. Food Eng. 61:557-560.

Dufour, E., G. Mazerolles, M. F. Devaux, G. Duboz, M. H. Duployer, and N. Mouhous Riou. 2000. Phase transition of triglycerides during semi-hard cheese ripening. Int. Dairy J. 10:81-93.

Frank, J. F., and G. S. Birth. 1982. Application of near infrared reflectance spectroscopy to cheese analysis. J. Dairy Sci. 65:1110-1116.

Haaland, D. M., and E. V. Thomas. 1988. Partial Least-Squares methods for spectral analyses. 1 . Relation to other quantitative calibration methods and the extraction of qualitative information. Anal. Chem. 60:1193-1202.

Hrushka, W. R. 1987. Data Analysis: Wavelength Selection Methods. Pages 35-55 in Near-Infrared Technology in the Agricultural and Food Industries. P. Williams and K. Norris, ed. American Association of Cereal Chemists Inc., St. Paul, MN.
Karoui, R., E. Dufour, L. Pillonel, D. Picque, T. Cattenoz, and J.-O. Bosset. 2004. Determining the geographic origin of Emmental cheeses produced during winter and summer using a technique based on the concatenation of MIR and fluorescence spectroscopic data. Eur. Food Res. Technol. 219:184-189.

Karoui, R., E. Dufour, L. Pillonel, E. Schaller, D. Picque, T. Cattenoz, and J.-O. Bosset. 2005. The potential of combined infrared and fluorescence spectroscopies as a method of determination of the geographic origin of Emmental cheeses. Int. Dairy J. 15:287-298.

Luginbühl, W. 2002. Evaluation of designed calibration samples for casein calibration in Fourier Transform Infrared analysis of milk. Lebensm. Wiss. Technol. 35:554-558.

Mazerolles, G., G. Duboz, and S. Hugot. 2000. Moisture and fat content determination in hard and semi-hard cheeses by near-infrared spectroscopy in transmission mode. Lait 80:371-379.

Martens, H., and T. Naes. 1989. Models for calibration. Chapter 3 in Multivariate Calibration. H. Martens and T. Naes, ed. John Wiley \& Sons Ltd, London, UK.

McQueen, D. H., R. Wilson, A. Kinnunen, and E. P. Jensen. 1995. Comparison of two infrared spectroscopic methods for cheese analysis. Talanta 42:2007-2015.

O'Sullivan, A., B. O'Connor, A. Kelly, and M. J. McGrath. 1999. The use of chemical and infrared methods for analysis of milk and dairy products. Int. J. Dairy Technol. 52:139-148.

Paradkar, M. M., and J. Irudayaraj. 2002. Determination of cholesterol in dairy products using infrared techniques: 1. FTIR spectroscopy. Int. J. Dairy Technol. 55:127-132.

Picque, D., T. Cattenoz, and G. Corrieu. 2002. Discrimination of Emmental cheeses by infrared spectroscopy. Milchwissenschaft 57:202-204.

Pierce, M. M., and R. L. Wehling. 1994. Comparison of sample handling and data treatment methods for determining moisture and fat in Cheddar cheese by near-infrared spectroscopy. J. Agric. Food Chem. 42:2831-2835.

Pike Technologies. 2005. ATR Theory and Applications. Applications Note 0402. PIKE Technologies, www.piketech.com

Pillonel, L., W. Luginbühl, D. Picque, E. Schaller, R. Tabacchi, and J. O. Bosset. 2003. Analytical methods for the determination of the geographic origin of Emmental cheese: Mid- and near-infrared spectroscopy. Eur. Food Res. Technol. 216:174-178.

Rodriguez-Otero, J. L., M. Hermida, and J. Centeno. 1997. Analysis of dairy products by near-infrared spectroscopy: A review. J. Agric. Food Chem. 45:2815-2819.

Rodriguez-Otero, J. L., M. Hermida, and A. Cepeda. 1995. Determination of fat, protein, and total solids in cheese by near-infrared reflectance spectroscopy. J. AOAC Int. 78:802-806.

Sivakesava, S., and J. Irudayaraj. 2002. Rapid determination of tetracycline in milk by FT-MIR and FT-NIR spectroscopy. J. Dairy Sci. 85:487-493.

Sorensen, L. K., M. Lund, and B. Juul. 2003. Accuracy of Fourier transform infrared spectrometry in determination of casein in dairy cows' milk. J. Dairy Res. 70:445-452.

Sorensen, L. K., and R. Jepsen. 1998. Comparison of near infrared spectroscopic techniques for determination of semi-hard cheese constituents. Milchwissenschaft 53:263-267.

Tsenkova, R., S. Atanassova, K. Toyoda, Y. Ozaki, K. Itoh, and T. Fearn. 1999. Near-infrared spectroscopy for dairy management: Measurement of unhomogenized milk composition. J. Dairy Sci. 82:2344-2351.

Udelhoven, T., D. Nauman, and J. Schmitt. 2000. Development of a Hierarchical classification system with artificial neural networks and FT-IR spectra for the identification of bacteria. Appl. Spectrosc. 54:1471-1479.

Wehling, R. L., and M. M. Pierce. 1988. Determination of moisture in Cheddar cheese by near infrared reflectance spectroscopy. J. AOAC 71:571-574.

Wittrup, C., and L. Nørgaard. 1998. Rapid near infrared spectroscopic screening of chemical parameters in semi-hard cheese using chemometrics. J. Dairy Sci. 81:1803-1809. 